\title{
PROBLEMS OF SPONTANEOUS AND GRAVITATIONAL BARYOGENESIS
}

\author{
Elena Arbuzova ${ }^{a}$ and Alexander Dolgov ${ }^{b}$ \\ ${ }^{a, b}$ Novosibirsk State University, Pirogova ul., 2, 630090, Novosibirsk, Russia \\ ${ }^{a}$ Dubna State University, Universitetskaya ul., 19, 141983, Dubna, Russia \\ ${ }^{b}$ ITEP, Bol. Cheremushkinsaya ul., 25, 117259, Moscow, Russia \\ Abstract. Spontaneous and closely related to it gravitational baryogenesis are \\ critically analyzed. It is shown that the coupling of the curvature scalar to baryonic \\ current, which induces nonzero baryonic asymmetry of the universe, simultaneously \\ leads to higher order gravitational equations, which have exponentially unstable \\ solutions. It is shown that this instability endangers the standard cosmology.
}

Different scenarios of baryogenesis are based, as a rule, on three well known Sakharov principles [1]: a) non-conservation of baryonic number; b) breaking of symmetry between particles and antiparticles; c) deviation from thermal equilibrium. For details see e.g. reviews 2]. However, none of these conditions is obligatory. Only one from three Sakharov principles, namely, non-conservation of baryons, is necessary for spontaneous baryogenesis (SBG) in its classical version [3, but this mechanism does not demand an explicit $\mathrm{C}$ and $\mathrm{CP}$ violation. It can proceed in thermal equilibrium and it is usually most efficient in thermal equilibrium.

The term "spontaneous" is related to spontaneous breaking of a global $U(1)$ symmetry, which ensures the conservation of the total baryonic number in the unbroken phase. When the symmetry is broken, the baryonic current becomes non-conserved and the Lagrangian density acquires the term

$$
\mathcal{L}_{S B G}=\left(\partial_{\mu} \theta\right) J_{B}^{\mu},
$$

$\theta$ is the (pseudo)Goldstone field and $J_{B}^{\mu}$ is the baryonic current of matter fields.

For a spatially homogeneous field $\theta=\theta(t)$ the Lagrangian (11) is reduced to $\mathcal{L}_{S B}=\dot{\theta} n_{B}$, where $n_{B} \equiv J_{B}^{0}$ is the baryonic number density of matter, so it is tempting to identify $(-\dot{\theta})$ with the baryonic chemical potential, $\mu_{B}$, of the corresponding system. As is argued in refs. 4, 5, such identification is questionable and depends upon the representation chosen for the fermionic fields, but still the scenario is operative and presents a beautiful possibility to create an excess of particles over antiparticles in the universe.

Subsequently the idea of gravitational baryogenesis (GBG) was put forward [6, where the scenario of SBG was modified by the introduction of the coupling of the baryonic current to the derivative of the curvature scalar $R$ :

$$
\mathcal{L}_{G B G}=\frac{f}{m_{0}^{2}}\left(\partial_{\mu} R\right) J_{B}^{\mu},
$$

\footnotetext{
${ }^{a}$ E-mail: arbuzova@uni-dubna.ru

${ }^{b}$ E-mail: dolgov@fe.infn.it
} 
where $m_{0}$ is a constant parameter with dimension of mass and $f$ is dimensionless coupling constant which is introduced for the arbitrariness of the sign.

GBG scenarios possess the same interesting and nice features of SBG, namely generation of cosmological asymmetry in thermal equilibrium without necessity of explicit $\mathrm{C}$ or $\mathrm{CP}$ violation in particle physics. However, an introduction of the derivative of the curvature scalar into the Lagrangian of the theory results in high order gravitational equations which are strongly unstable. The effects of this instability may drastically distort not only the usual cosmological history, but also the standard Newtonian gravitational dynamics. We discovered such instability for scalar baryons [7] and found similar effect for the more usual spin one-half baryons (quarks) [8].

Let us start from the model where baryonic number is carried by scalar field $\phi$ with potential $U\left(\phi, \phi^{*}\right)$. The action of the scalar model has the form:

$A=\int d^{4} x \sqrt{-g}\left[\frac{m_{P l}^{2}}{16 \pi} R+\frac{1}{m_{0}^{2}}\left(\partial_{\mu} R\right) J^{\mu}-g^{\mu \nu} \partial_{\mu} \phi \partial_{\nu} \phi^{*}+U\left(\phi, \phi^{*}\right)\right]-A_{m}$,

where $m_{P l}=1.22 \cdot 10^{19} \mathrm{GeV}$ is the Planck mass, $A_{m}$ is the matter action, $J^{\mu}=g^{\mu \nu} J_{\nu}$, and $g^{\mu \nu}$ is the metric tensor of the background space-time. We assume that initially the metric has the usual GR form and study the emergence of the corrections due to the instability described below.

In the homogeneous case the equation for the curvature scalar in the FRW metric takes the form:

$\frac{m_{P l}^{2}}{16 \pi} R+\frac{q^{2}}{6 m_{0}^{4}}\left(R+3 \partial_{t}^{2}+9 H \partial_{t}\right)\left[(\ddot{R}+3 H \dot{R}) T^{2}\right]+\frac{1}{m_{0}^{2}} \dot{R}\left\langle J^{0}\right\rangle=-\frac{T^{(t o t)}}{2}$,

where $\left\langle J^{0}\right\rangle$ is the thermal average value of the baryonic number density of $\phi$, $q$ is the baryonic number of $\phi, H=\dot{a} / a$ is the Hubble parameter, and $T^{(t o t)}$ is the trace of the energy-momentum tensor of matter including contribution from the $\phi$-field. In the homogeneous and isotropic cosmological plasma

$$
T^{(t o t)}=\rho-3 P,
$$

where $\rho$ and $P$ are respectively the energy density and the pressure of the plasma. For relativistic plasma $\rho=\pi^{2} g_{*} T^{4} / 30$ with $T$ and $g_{*}$ being the plasma temperature and the number of particle species in the plasma. The Hubble parameter is expressed through $\rho$ as $H^{2}=8 \pi \rho /\left(3 m_{P l}^{2}\right) \sim T^{4} / m_{P l}^{2}$.

Keeping only the linear in $R$ terms and neglecting higher powers of $R$, such as $R^{2}$ or $H R$, we obtain the linear fourth order differential equation:

$$
\frac{d^{4} R}{d t^{4}}+\mu^{4} R=-\frac{1}{2} T^{(t o t)}, \text { where } \mu^{4}=\frac{m_{P l}^{2} m_{0}^{4}}{8 \pi q^{2} T^{2}} .
$$

The homogeneous part of this equation has exponential solutions $R \sim \exp (\lambda t)$ with $\lambda=|\mu| \exp (i \pi / 4+i \pi n / 2)$, where $n=0,1,2,3$. There are two solutions 
with positive real parts of $\lambda$. This indicates that the curvature scalar is exponentially unstable with respect to small perturbations, so $R$ should rise exponentially fast with time and quickly oscillate around this rising function.

Now we need to check if the characteristic rate of the perturbation explosion is indeed much larger than the rate of the universe expansion, that is:

$$
(\operatorname{Re} \lambda)^{4}>H^{4}=\left(\frac{8 \pi \rho}{3 m_{P l}^{2}}\right)^{2}=\frac{16 \pi^{6} g_{*}^{2}}{2025} \frac{T^{8}}{m_{P l}^{4}},
$$

where $\rho=\pi^{2} g_{*} T^{4} / 30$ is the energy density of the primeval plasma at temperature $T$ and $g_{*} \sim 10-100$ is the number of relativistic degrees of freedom in the plasma. This condition is fulfilled if

$$
\frac{2025}{2^{9} \pi^{7} q^{2} g_{*}^{2}} \frac{m_{P l}^{6} m_{0}^{4}}{T^{10}}>1,
$$

or, roughly speaking, if $T \leq m_{P l}^{3 / 5} m_{0}^{2 / 5}$. At these temperatures the instability is quickly developed and the standard cosmology would be destroyed.

Let us now generalize results, obtained for scalar baryons, to realistic fermions. We start from the action in the form

$$
\begin{aligned}
A & =\int d^{4} x \sqrt{-g}\left[\frac{m_{P l}^{2}}{16 \pi} R-\mathcal{L}_{m}\right] \\
\mathcal{L}_{m} & =\frac{i}{2}\left(\bar{Q} \gamma^{\mu} \nabla_{\mu} Q-\nabla_{\mu} \bar{Q} \gamma^{\mu} Q\right)-m_{Q} \bar{Q} Q \\
& +\frac{i}{2}\left(\bar{L} \gamma^{\mu} \nabla_{\mu} L-\nabla_{\mu} \bar{L} \gamma^{\mu} L\right)-m_{L} \bar{L} L \\
& +\frac{g}{m_{X}^{2}}\left[\left(\bar{Q} Q^{c}\right)(\bar{Q} L)+\left(\bar{Q}^{c} Q\right)(\bar{L} Q)\right]+\frac{f}{m_{0}^{2}}\left(\partial_{\mu} R\right) J^{\mu}+\mathcal{L}_{\text {other }}
\end{aligned}
$$

where $Q$ is the quark (or quark-like) field with non-zero baryonic number, $L$ is another fermionic field (lepton), $\nabla_{\mu}$ is the covariant derivative of Dirac fermion in tetrad formalism, $J^{\mu}=\bar{Q} \gamma^{\mu} Q$ is the quark current with $\gamma^{\mu}$ being the curved space gamma-matrices, $\mathcal{L}_{\text {other }}$ describes all other forms of matter. The fourfermion interaction between quarks and leptons is introduced to ensure the necessary non-conservation of the baryon number with $m_{X}$ being a constant parameter with dimension of mass and $g$ being a dimensionless coupling constant. In grand unified theories $m_{X}$ may be of the order of $10^{14}-10^{15} \mathrm{GeV}$.

Varying the action (9) over metric, $g^{\mu \nu}$, and taking trace with respect to $\mu$ and $\nu$, we obtain the following equation of motion for the curvature scalar:

$$
\begin{aligned}
-\frac{m_{P l}^{2}}{8 \pi} R & =m_{Q} \bar{Q} Q+m_{L} \bar{L} L+\frac{2 g}{m_{X}^{2}}\left[\left(\bar{Q} Q^{c}\right)(\bar{Q} L)+\left(\bar{Q}^{c} Q\right)(\bar{L} Q)\right] \\
& -\frac{2 f}{m_{0}^{2}}\left(R+3 D^{2}\right) D_{\alpha} J^{\alpha}+T_{\text {other }}
\end{aligned}
$$


where $T_{\text {other }}$ is the trace of the energy momentum tensor of all other fields. At relativistic stage, when masses are negligible, we can take $T_{\text {other }}=0$. The average expectation value of the interaction term proportional to $g$ is also small, so the contribution of all matter fields may be neglected.

We used the kinetic equation, which leads to an explicit dependence on $R$ of the current divergence, $D_{\alpha} J^{\alpha}$, if the current is not conserved. As a result we obtain 4th order differential equation for $R$ :

$$
\frac{d^{4} R}{d t^{4}}=\lambda^{4} R, \text { where } \lambda^{4}=\frac{5 m_{P l}^{2} m_{0}^{4}}{36 \pi g_{s} B_{q} f^{2} T^{2}} .
$$

Here $g_{s}$ and $B_{q}$ are respectively the number of the spin states and the baryonic number of quarks. Deriving this equation we neglected the Hubble parameter factor in comparison with time derivatives of $R$.

Evidently equation (11) has extremely unstable solution with instability time by far shorter than the cosmological time. This instability would lead to an explosive rise of $R$, which may possibly be terminated by the nonlinear terms proportional to the product of $H$ to lower derivatives of $R$. Correspondingly one may expect stabilization when $H R \sim \dot{R}$, i.e. $H \sim \lambda$. Since $\dot{H}+2 H^{2}=-R / 6$, $H$ would also exponentially rise together with $R, H \sim \exp (\lambda t)$ and $\lambda H \sim R$. Thus stabilization may take place at $R \sim \lambda^{2} \sim m_{P l} m_{0}^{2} / T$. This result should be compared with the normal General Relativity value $R_{G R} \sim T_{\text {matter }} / m_{P l}^{2}$, where $T_{\text {matter }}$ is the trace of the energy-momentum tensor of matter.

\section{Acknowledgments}

This work was supported by the RSF Grant N 16-12-10037.

[1] A.D. Sakharov, Pis'ma ZhETF 5 (1967) 32.

[2] A.D.Dolgov, Phys. Repts 222 (1992) No. 6; A.D. Dolgov, Surveys in High Energy Physics, 13 (1998) 83; V.A. Rubakov, M.E. Shaposhnikov, Usp. Fiz. Nauk, 166 (1996) 493.

[3] A. Cohen, D. Kaplan, Phys. Lett. B 199, (1987) 251; A. Cohen, D. Kaplan, Nucl.Phys. B 308 (1988) 913; A. G. Cohen, D.B. Kaplan, A.E. Nelson, Phys.Lett. B 263 (1991) 86; A. G. Cohen, D.B. Kaplan, A.E. Nelson, Phys.Lett. B 336 (1994) 41.

[4] A.D. Dolgov, K. Freese, Phys.Rev. D 51 (1995) 2693-2702; A.D. Dolgov, K. Freese, R. Rangarajan, M. Srednicki, Phys. Rev. D 56 (1997) 6155.

[5] E.V. Arbuzova, A.D. Dolgov, V.A. Novikov, Phys. Rev. D 94 (2016) 123501.

[6] H. Davoudiasl, R. Kitano, G. D. Kribs, H. Murayama, P. J. Steinhardt, Phys. Rev. Lett. 93 (2004) 201301.

[7] E.V. Arbuzova, A. D. Dolgov, Phys. Lett. B 769 (2017) 171.

[8] E.V. Arbuzova, A. D. Dolgov, JCAP 1706 (2017) 001. 